\title{
Abdominal CT in COVID-19 patients: incidence, indications, and findings
}

\author{
Daniel T. Barkmeier ${ }^{1}\left[\right.$ D Erica B. Stein ${ }^{1}$ (D) Katherine Bojicic ${ }^{1} \cdot$ Bamidele Otemuyiwa $^{1} \cdot$ Dharshan Vummidi $^{1}$. \\ Aamer Chughtai ${ }^{1}$. James H. Ellis ${ }^{1}$ (1)
}

Received: 14 July 2020 / Revised: 31 August 2020 / Accepted: 3 September 2020 / Published online: 19 September 2020

๑) Springer Science+Business Media, LLC, part of Springer Nature 2020

\begin{abstract}
Purpose The purpose of this study was to evaluate the frequency, indications, and findings of abdominal CTs ordered in the initial evaluation of patients who had a positive COVID-19 test performed in our institution.

Methods Retrospective chart review was performed on all patients who had a positive test for COVID-19 performed at a single quaternary care center from $1 / 20 / 2020$ through 5/8/2020. In a subset of patients undergoing abdominal CT as part of the initial evaluation, the demographics, suspected COVID-19 status at the time of scan, presenting complaints, and abdominal CT findings were recorded. Cardiothoracic radiologists reviewed and scored the visualized lung bases for the likelihood of COVID-19.

Results Only 43 (4.1\%) of 1057 COVID-19 patients presented with abdominal complaints sufficient to warrant an abdominal CT. Of these 43 patients, the vast majority $(39,91 \%)$ were known or suspected to have COVID-19 at the time of the scan. Most $(27 / 43,63 \%)$ scans showed no acute abdominal abnormality, and those that were positive did not share a discernable pattern of abnormalities. Lung base abnormalities were common, and there was moderate inter-reviewer reliability.

Conclusion A minority of COVID-19 patients present with abdominal complaints sufficient to warrant a dedicated CT of the abdomen, and most of these studies will be negative or have abdominal findings not associated with COVID-19. Appropriate lung base findings are a more consistent indication of COVID-19 infection than abdominal findings.
\end{abstract}

Keywords COVID-19 - Coronavirus · Severe acute respiratory syndrome coronavirus $2 \cdot$ Abdomen · Lung diseases · Tomography $\cdot \mathrm{X}$-ray computed

\section{Introduction}

The majority of severe morbidity and mortality from the pandemic novel coronavirus COVID-19 (SARS-CoV-2) disease have been pulmonary $[1,2]$ and imaging abnormalities of the lungs have been a hallmark of the disease [3-6]. The pulmonary manifestations have tended to overshadow the abdominal symptoms of the disease, even though some reports [7] have indicated that as many as $57 \%$ of patients with low-severity COVID-19 disease have abdominal complaints alone or in combination with pulmonary symptoms. If abdominal CT is performed, the radiologist may be the

Daniel T. Barkmeier

dbarkmei@med.umich.edu

1 Department of Radiology, Michigan Medicine, University of Michigan, 1500 E. Medical Center Drive, Ann Arbor, MI 48109-5030, USA first to suggest COVID-19 when imaging of the lung bases show typical findings of COVID-19 pulmonary disease [8-10]. In other patients, the diagnosis of COVID-19 may already be known or suspected, but abdominal symptoms are of sufficient severity to result in requests for abdominal CT.

Radiologists are aware of the possible pulmonary findings on CT scans of the thorax in COVID-19 patients, including ground-glass opacities and consolidation. However, radiologists may be less familiar with the frequency of abdominal symptoms in these patients and that abdominal complaints may be present in the absence of clinical pulmonary disease. Findings at the lung bases in abdominal CT scans may be the first hint of novel coronavirus disease [8-10].

We performed a retrospective review of patients who had a positive test for COVID-19 performed at our quaternary care institution to determine the frequency with which abdominal CT scans were ordered in their initial workup. In addition, we assessed the clinical indications for the scans 
and whether intra-abdominal pathology was uncovered by the scans. The medical records were reviewed to determine whether the patient was known or suspected to have COVID19 disease as a measure of whether lung findings might be the first indication of the diagnosis. Finally, we reviewed the imaging of the lung bases to determine the frequency of findings suspicious for novel coronavirus infection.

\section{Methods}

Institutional review board approval was obtained, and the requirement for informed consent was waived for this Health Insurance Portability and Accountability Act-compliant retrospective investigation.

The electronic medical record was searched using a tool called EMERSE [11] to identify patients who had a positive test at our institution for the novel coronavirus during the study period from January 1, 2020 through May 8, 2020. Transfer patients with the diagnosis of COVID-19 who received initial work-up and COVID-19 testing at another institution were excluded, as many of these patients already had known, significant pulmonary disease as the indication for their transfer.

The same EMERSE tool was used to identify the subset of patients who underwent an abdominal CT during the study time period. Demographic data including age, sex, race, body mass index (BMI), and history of diabetes mellitus were exported for both groups of patients (those who had an abdominal CT and those who did not), using a locally provided user-accessible interface into the institutional electronic medical record data, DataDirect. Manual chart review (authors KB and BO) was performed to identify which patients had an abdominal $\mathrm{CT}$ related to their initial presentation, as opposed to an unrelated CT examination that happened to occur during the study time period. Additional data pertaining to each patient's presenting symptoms, CT requisition information, clinical COVID-19 status at presentation, reason for admission, and CT findings in the original radiology report were recorded as part of the manual chart review. Patients who had a CT unrelated to their initial presentation were moved to the "No CT" group. The CT examinations were performed on MDCT scanners (GE Healthcare and Siemens). Abdominal CTs (including lung bases) were acquired at $0.625 \mathrm{~mm}$ slice thickness and reconstructed as $5 \mathrm{~mm}$ axial slices, with the exception of a single pancreatic scan reconstructed as $2.5 \mathrm{~mm}$ axial slices.

At our institution, the thoracic and abdominal portions of an examination are reported separately by subspecialty radiologists, so the lung bases were often not described in abdominal CT reports for patients who had a concurrent chest CT. Therefore, to determine how often typical findings for COVID-19 were present in the lung bases of all study patients, the visible lung bases of each relevant CT were reviewed independently by two subspecialty-trained cardiothoracic radiologists (DV and AC, with 10 and 16 years of experience) and scored as typical, indeterminate, or atypical for COVID-19, or negative for pneumonia, based on the RSNA-STR-ACR system (Radiological Society of North America-Society of Thoracic Radiology-American College of Radiology) [12].

Continuous variables were summarized with means \pm standard deviations and compared between groups with a $t$ test. Categorical variables were presented as counts (\%) and compared with the Chi-square test. Reviewer agreement was evaluated with Cohen's kappa, using linear weighting due to the ordinal nature of the data. All statistical analyses were performed in Excel (Microsoft, 2016 version).

\section{Results}

Our study population consisted of 1057 patients who tested positive for COVID-19 at our institution. Of these, 43 (4.1\%) underwent an abdominal $\mathrm{CT}$ as part of their initial presentation. Of the 43 abdominal CT scans performed, 26 were performed with intravenous contrast and 17 without; 12 scans were performed with the administration of oral contrast, one of which was neutral contrast for a pancreatic scan. Compared to patients who did not receive an abdominal CT, the group who underwent CT were significantly older (mean $64.5 \pm 17.3$ years versus $52.8 \pm 19.0$ years; $p=0.00009$ ) and more likely to have diabetes mellitus (65\% versus $29 \%$; $p=0.0000003$ ). There was borderline difference in the distribution of races between the two groups $(p=0.047)$, with African Americans over-represented in the group undergoing abdominal CT. The groups did not significantly differ in sex or body mass index (Table 1).

The majority of patients imaged with abdominal CT presented to the Emergency Department $(31 / 43,72 \%)$ with the rest comprised transfer, clinic, or skilled nursing facility patients. Most $(40 / 43,93 \%)$ were subsequently admitted to the hospital. Reasons for admission most commonly included respiratory failure/hypoxia $(20 / 40,50 \%)$, acute kidney injury $(12 / 40,30 \%)$, altered mental status (11/40, $27.5 \%)$, and sepsis/infection (10/40, 25\%); most patients $(25 / 40,63 \%)$ had more than one reason for admission.

At the time the abdominal CT was ordered, 21 (49\%) patients had already tested positive for COVID-19, and 18 (42\%) were suspected by the clinical team to have the disease. Despite this prevalence, COVID-19 was mentioned by the ordering providers in only $4(10 \%)$ of the $39 \mathrm{CT}$ requisitions in patients with known or suspected COVID-19. In only $4 / 43$ (9\%) patients was COVID-19 not suspected at the time of CT. In two of these patients, the reports of the abdominal CT scans referred to patchy lung base opacities 
Table 1 Demographic data of patients testing positive for COVID-19 infection who did or did not undergo abdominal CT scanning

\begin{tabular}{llll}
\hline & $\begin{array}{l}\text { Patients undergoing abdominal } \\
\text { CT scans }(\mathrm{N}=43)\end{array}$ & $\begin{array}{l}\text { Patients without abdominal CT } \\
\text { scans }(\mathrm{N}=1014)\end{array}$ & $p$ value \\
\hline Age (years) & & & 0.00009 \\
Mean \pm SD & $64.5 \pm 17.3$ & $52.8 \pm 19.0$ & \\
Range & $26-96$ & $18-101$ & 0.2 \\
Sex $(\%)$ & & $465(46)$ & \\
Male & $24(56)$ & $549(54)$ & 0.047 \\
Female & $19(44)$ & & \\
Race $(\%)$ & & $467(46)$ & \\
Caucasian & $16(37)$ & $365(36)$ & \\
African American & $24(56)$ & $56(6)$ & \\
Asian & $1(2)$ & $126(12)$ & \\
Other/unknown & $2(5)$ & $31.4 \pm 8.5$ & \\
BMI (kg/m $\left.{ }^{2}\right)$ & & $11-61$ & 0.097 \\
Mean \pm SD & $29.4 \pm 7.9$ & & \\
Range & $16-51$ & $291(29)$ & \\
Diabetes mellitus $(\%)$ & & & \\
Patients & $28(65)$ & &
\end{tabular}

that could represent viral pneumonia, and the abdominal CT led to testing/diagnosis. In a third patient, the report of a concurrent chest CT described multifocal areas of patchy ground-glass opacity and raised the possibility of infection. In the fourth patient, a concurrent chest CT was reported as negative; the patient had been found unresponsive after an unwitnessed fall and the CT scans were obtained to evaluate for traumatic injury whereas the positive COVID-19 test was obtained as part of a general workup to exclude infection.

The most common presenting clinical complaints in the 43 patients that underwent abdominal CT were fever in 18 (42\%), shortness of breath in $17(40 \%)$, cough in $16(37 \%)$ and altered mental status in $12(28 \%)$ (Table 2). The most common indications for abdominal CT listed on the imaging requisitions were abdominal pain in $22(51 \%)$, fever in eight (19\%), and abdominal infection in seven (16\%).

In $27(63 \%)$ of 43 examinations, the abdominal CT scans showed no acute abnormalities. In seven (16\%) scans, the interpreting radiologists reported abnormalities in the luminal gastrointestinal tract, all of which were inflammatory or infectious in nature. Two cases were positive for colitis, one of which demonstrated pneumatosis. Additional pathologies included one case each of gastritis, equivocal findings of appendicitis, concurrent colonic diverticulitis and perforated marginal ulcer at the gastrojejunal anastomosis in a gastric bypass patient, stercoral colitis, and concurrent stercoral colitis and diverticulitis. Abnormalities of the urinary tract were reported in five (12\%) scans: two cases of cystitis, and one case each of pyelonephritis, renal abscess, and obstructing ureteropelvic junction stone. Other conditions reported were one case each of liver abscess, progression of hepatic metastases from known gastric carcinoid tumor, pancreatitis, new small splenic infarct in a patient with prior imaging suggesting pancreatic and renal cell cancers, and a simple fluid collection in the right thigh.

Two subspecialty-trained and practicing cardiothoracic radiologists reviewed the visualized lung bases in the cohort of patients who underwent abdominal CT. One patient had insufficient lung parenchyma visible to evaluate, so this patient was removed from consideration, leaving 42 abdominal CT scans with sufficient lung parenchyma to review. The majority of these patients $(32 / 42,76.2 \%)$ had abnormality beyond atelectasis in the lung bases, most commonly ground-glass opacities (Fig. 1). By the RSNA-STR-ACR consensus reporting standards [12], a large group of patients had findings "Typical” for COVID-19 pneumonia (Reviewer 1, 12 [29\%]; Reviewer 2, 20 [48\%]), fewer had "Indeterminate" findings (10 [24\%]; 7 [17\%]), a minority had findings "Atypical" for COVID-19 pneumonia (8 [19\%]; 5 [12\%]), and about a fourth (12 [29\%]; 10 [24\%]) had no evidence of pneumonia. In 9 (21\%) of the 42 patients, both cardiothoracic radiologists agreed that no pneumonia was present. Despite limitations noted by the cardiothoracic radiologists, including thicker $5 \mathrm{~mm}$ sections, respiratory motion, and limited visible lung parenchyma, inter-reviewer agreement was moderate, with a weighted kappa value of 0.43 .

\section{Discussion}

Presentation of novel coronavirus patients with abdominal complaints is a known phenomenon [7, 13] that has been overshadowed by the substantial morbidity and mortality of COVID-19 patients who develop respiratory distress 
Table 2 Clinical presenting complaints and indications for abdominal $\mathrm{CT}$ in 43 patients testing positive for COVID-19 infection

\begin{tabular}{lcc}
\hline Condition & $\begin{array}{l}\text { Listed as a } \\
\text { presenting } \\
\text { complaint }\end{array}$ & $\begin{array}{l}\text { Listed as a requisition } \\
\text { indication for abdominal } \\
\text { CT }\end{array}$ \\
\hline Fever & 18 & 8 \\
Shortness of breath & 17 & \\
Cough & 16 & 1 \\
Altered mental status & $12^{\mathrm{a}}$ & \\
Myalgias & 8 & \\
Fatigue/weakness & 7 & 22 \\
Abdominal pain & 7 & \\
Diarrhea & 7 & 5 \\
Trauma & 5 & \\
Vomiting & 5 & \\
Nausea & 2 & \\
Chills & 2 & 3 \\
Headache & 2 & 3 \\
Abdominal infection & & 3 \\
Abdominal distention & & \\
Abdominal neoplasm & & \\
Urinary tract stone & & \\
$\quad$ disease & & \\
Miscellaneous & $8^{\mathrm{b}}$ & \\
\hline
\end{tabular}

Table ordered by frequency of presenting complaint. Clinical presenting complaints abstracted from the electronic medical record. Indications for abdominal CT obtained from the electronic requisitions. Conditions are summarized rather than verbatim text. Totals exceed 43 because patients had multiple conditions

${ }^{\mathrm{a}}$ One patient presented with a stroke

${ }^{b}$ Miscellaneous presenting complaints were hematuria and urinary urgency, urinary incontinence, renal failure, rhinorrhea, chest pain, vaginal bleeding, hematochezia, and anorexia with weight loss

${ }^{\mathrm{c}}$ Miscellaneous indications for abdominal CT were renal failure, hematuria, and hypotension.

[1]. Radiologists not only need to be aware that COVID-19 patients may have abdominal symptoms, but also that such patients may have no or only mild pulmonary symptoms [7, 13], and may not undergo dedicated thoracic imaging. The majority of these patients will have no or nonspecific findings on abdominal $\mathrm{CT}$, though of course positive findings on abdominal CT does not exclude COVID-19 disease.

Positive intra-abdominal findings in our study were similar to our pre-pandemic routine patients evaluated for abdominal pain in the emergency department, with no distinct patterns emerging that seem specific for COVID-19. An intra-abdominal abnormality specific for COVID-19 infection has yet to be determined. As such, it is reasonable to conclude that currently the characteristic pulmonary findings of COVID-19, if present at the lung bases of an abdominal CT, should be the sole abdominal CT abnormality suggesting the possibility of novel coronavirus infection.
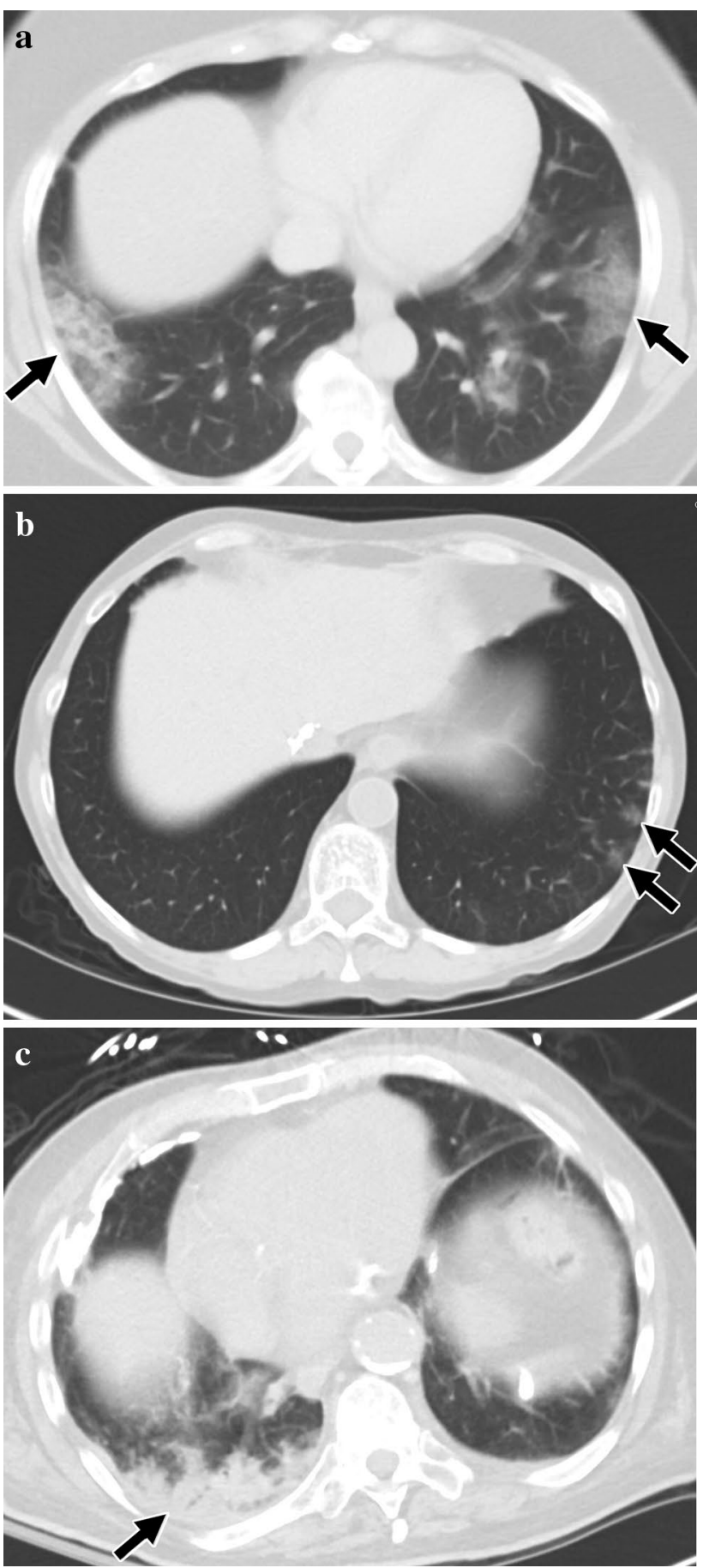

Fig. 1 Axial images of the lung bases in lung windows from three different patients showing examples of the scoring by two subspecialty cardiothoracic radiologists. a Typical appearance for COVID19 pneumonia. There are large bilateral, subpleural regions of ground-glass opacity (arrows), with visible intralobular lines ("crazypaving"). b Indeterminate for COVID-19 pneumonia. There are only a few unilateral ground-glass opacities (arrows) in a single lobe. c Atypical appearance for COVID-19 pneumonia. There is an isolated consolidation (arrow) in the base of the right lower lobe 
The characteristic pulmonary findings of COVID-19 at the lung bases may be present in a patient not yet suspected to be infected, or serve as supporting evidence in a patient under investigation for novel coronavirus infection.

Our results differ from those of Bhayana et al. [14] who reviewed all the abdominal imaging in 412 patients admitted to their hospital who tested positive for COVID-19, and found bowel wall abnormalities (primarily bowel wall thickening) in $31 \%$, and pneumatosis intestinalis or portal venous gas in almost $10 \%$, of the 42 abdominal CT scans performed. We found a similar percentage $(35 \%)$ of patients with abdominal abnormalities on CT, but bowel wall changes were uncommon. Only three (7\%) patients showed CT findings of a nonspecific enteritis, with pneumatosis intestinalis present on one scan, and no findings of portal venous gas. Our results are more similar to Goldberg-Stein et al. [15] who also found that gastrointestinal tract findings were the most common category of abnormality on abdominal CTs (31.2\%), but with wall thickening being less common (15\%) and no reported cases of pneumatosis or portal venous gas. Another study [9] of 23 patients with lung base findings suspicious for COVID-19 infection reported a variety of typical findings characteristic of pre-COVID-19 abdominal pain diagnoses, with no abnormalities of the luminal gastrointestinal tract. A potential reason for the differences among the studies is that Bhayana et al. may have included patients later in the course of their disease, whereas our study and those of Goldberg-Stein et al. [15] and Dane et al. [9] reviewed CT scans obtained around the time of COVID-19 diagnosis.

In our study of 42 COVID-19 patients undergoing abdominal CT with sufficient visualized lung bases to evaluate, nine (21\%) had normal lung bases as interpreted by both subspecialty cardiothoracic radiologists and 33 (79\%) had abnormal lung bases as interpreted by one or both cardiothoracic radiologists, when using the RSNA-STR-ACR criteria. Findings suggestive of either COVID-19 specifically, or viral pneumonia more generally [12] were noted in 22 (52\%) patients by Reviewer 1 and 27 (64\%) patients by Reviewer 2. Cohen's Kappa with linear weighting was 0.43 , which is at the low end of the moderate agreement range, but considered acceptable, given the limitations of thick $(5 \mathrm{~mm})$ sections, respiratory motion, and limited lung parenchyma visible to evaluate.

Most abdominal CT scans will include a portion of the lung bases, although there may be some exceptions depending on local techniques. Pre-pandemic reports have assessed the value of close examination of the lung bases for clinically significant abnormalities [16]. Our study, and others [8-10, 15], show that lung base abnormalities on abdominal CT may now include findings that suggest a diagnosis of COVID-19 disease in a substantial percentage of cases.

How often these lung base findings on abdominal CT are the first indication that a patient has COVID-19 disease is not clear. This is multifaceted and could depend on whether the COVID-19 infection was incidental to a symptomatic abdominal process, what the prevalence of COVID-19 spread was in the community, and the method for assessing whether COVID-19 was clinically suspected prior to the discovery of the lung base findings at CT. Dane et al. [9] reported 23 patients over a ten-day period whose abdominal CT scans had unanticipated findings at the lung bases suspicious for COVID-19, most of whom eventually returned positive laboratory tests for COVID-19 disease. However, the precise manner in which it was determined that there was no initial clinical suspicion for COVID-19 was not specifically stated. Vu et al. [8] and Siegel et al. [10] each reported a case series of three patients undergoing abdominal CT scans where the diagnosis of COVID-19 was first suspected based on the CT reports of lung base findings, but no data were supplied on the number of abdominal CT scans from which these cases were selected. In our study, review of the electronic medical record showed that in 43 patients with COVID-19 disease who underwent abdominal CT scans, the diagnosis of COVID-19 was already under consideration in 39 patients. In the four patients where COVID-19 was not suspected by the referring clinical service, the original CT reports raised the possibility of viral pneumonia in three, and the fourth was negative for pneumonia. In the 39 patients in whom COVID-19 was already under clinical consideration, only four of the CT requisitions mentioned COVID-19, suggesting that assessing the stated indications on examination requisitions may not be a reasonable way to assess pre-CTscan clinical suspicion for COVID-19 disease.

There are several limitations to our study. Compared to the large number of COVID-19 infected patients nationwide and worldwide, our study of 1057 patients, 43 of whom underwent abdominal CT scanning, is a small sample. Further, it is possible that our sample is biased by coming from an academic quaternary care institution, although this is tempered by our provision of substantial primary and secondary care within our local area. We chose to only include patients who had a positive COVID19 test at our institution in an attempt to have a more homogeneous sample and one in which transfer patients with long-standing complicated disease were excluded. However, in doing so, we may have excluded some patients who were rapidly transferred and may have arrived before the test results were known or who had not undergone any substantial workup at a previous institution other than having the test for novel coronavirus obtained. In addition, this methodology resulted in patients being scanned early in their disease progression; abdominal CT findings might be different later in patients' COVID-19 course. We did not prospectively ask healthcare providers about their rationale for requesting abdominal $\mathrm{CT}$ or about their suspicion for novel coronavirus disease in the patients 
that they referred for abdominal CT, and as a result, our data concerning how often abdominal CT was the first test to raise the suspicion of COVID-19 is subject to bias. Regardless, the exact percentages of COVID-19 patients who undergo abdominal CT, and who have abdominal or lung base findings, is not as important as the recognition that COVID-19 may present in less than typical ways and that abdominal CT may be one of the initial imaging tests. Finally, our cardiothoracic radiologists were not blinded to the COVID-19 status of the patients whose CT scans they reviewed to assess the lung bases, which could potentially bias them toward reporting lung findings as more typical of COVID-19.

In summary, abdominal CT is requested in a small number (about 4\%) of COVID-19-positive patients at presentation. Most of these patients had typical respiratory complaints, which when combined with clinical awareness about the disease, resulted in few opportunities for our radiologists to be the first to suggest the disease based on lung findings on abdominal CT. Nevertheless, lung findings on CT scans did suggest COVID-19 in three patients in whom the disease was not previously suspected, representing $7 \%$ of the abdominal CT scans in our study but less than $0.3 \%$ of all COVID-19 patients at presentation. Most (63\%) of our abdominal CT scans showed no acute abdominal abnormalities. While there are no abdomen CT findings specific for the disease, enteritis has been associated with COVID-19 disease, including enteritis accompanied by ischemia. We found only one (2\%) case of pneumatosis intestinalis and two $(5 \%)$ cases of nonspecific bowel inflammation, which may have been COVID-19 related or may have been coincidental. Most of our abdominal CT diagnoses were typical pre-pandemic diagnoses that have not been reported to be associated with COVID-19. Finally, we found that suspicion of COVID-19 is rarely communicated to the radiologist through the stated indications on the requisitions for abdominal CT scans, which suggests the need for alternate methods to assess clinical suspicion for COVID-19 disease if that information is desired for study interpretation or research.

\begin{abstract}
Author contributions All authors contributed to the study conception and design. Material preparation, data collection and analysis were performed by DB, EBS, KB, BO, AC, DV, JHE. The first draft of the manuscript was written by JHE, subsequent drafts by DB and EBS, and all authors commented on previous versions of the manuscript. All authors read and approved the final manuscript.
\end{abstract}

Funding No external funding.

\section{Compliance with ethical standards}

Conflict of interest The authors declare that they have no conflict of interest.
Consent for publication All authors consent to publication. No other consents were required or sought.

Ethical approval This retrospective chart review study involving human participants was in accordance with the ethical standards of the institutional research committee and with the 1964 Helsinki Declaration and its later amendments or comparable ethical standards. The Human Investigation Committee (IRB) approved this study (HUM00180779). The requirement for informed consent was waived for this retrospective investigation.

Informed consent Informed consent was not sought. The requirement for informed consent was waived by the local institutional review board approval.

\section{References}

1. Guan WJ, Ni ZY, Hu Y, Liang WH, Ou CQ, He JX, Liu L, Shan H, Lei CL, Hui DSC, Du B, Li LJ, Zeng G, Yuen KY, Chen RC, Tang CL, Wang T, Chen PY, Xiang J, Li SY, Wang JL, Liang ZJ, Peng YX, Wei L, Liu Y, Hu YH, Peng P, Wang JM, Liu JY, Chen Z, Li G, Zheng ZJ, Qiu SQ, Luo J, Ye CJ, Zhu SY, Zhong NS, China Medical Treatment Expert Group for C (2020) Clinical Characteristics of Coronavirus Disease 2019 in China. N Engl J Med 382 (18):1708-1720. https://doi.org/10.1056/nejmoa2002032

2. Huang G, Gong T, Wang G, Wang J, Guo X, Cai E, Li S, Li X, Yu Y, Lin L (2020) Timely Diagnosis and Treatment Shortens the Time to Resolution of Coronavirus Disease (COVID-19) Pneumonia and Lowers the Highest and Last CT Scores From Sequential Chest CT. AJR Am J Roentgenol:1-7. https://doi.org/10.2214/ ajr.20.23078

3. Bernheim A, Mei X, Huang M, Yang Y, Fayad ZA, Zhang N, Diao K, Lin B, Zhu X, Li K, Li S, Shan H, Jacobi A, Chung M (2020) Chest CT Findings in Coronavirus Disease-19 (COVID-19): Relationship to Duration of Infection. Radiology 295 (3):200463. https ://doi.org/10.1148/radiol.2020200463

4. Chung M, Bernheim A, Mei X, Zhang N, Huang M, Zeng X, Cui J, Xu W, Yang Y, Fayad ZA, Jacobi A, Li K, Li S, Shan H (2020) CT Imaging Features of 2019 Novel Coronavirus (2019-nCoV). Radiology 295 (1):202-207. https://doi.org/10.1148/radiol.20202 00230

5. Lei J, Li J, Li X, Qi X (2020) CT Imaging of the 2019 Novel Coronavirus (2019-nCoV) Pneumonia. Radiology 295 (1):18. https:// doi.org/10.1148/radiol.2020200236

6. Zhou S, Wang Y, Zhu T, Xia L (2020) CT Features of Coronavirus Disease 2019 (COVID-19) Pneumonia in 62 Patients in Wuhan, China. AJR Am J Roentgenol 214 (6):1287-1294. https://doi. org/10.2214/ajr.20.22975

7. Han C, Duan C, Zhang S, Spiegel B, Shi H, Wang W, Zhang L, Lin R, Liu J, Ding Z, Hou X (2020) Digestive Symptoms in COVID-19 Patients With Mild Disease Severity: Clinical Presentation, Stool Viral RNA Testing, and Outcomes. Am J Gastroenterol 115 (6):916-923. https://doi.org/10.14309/ajg.0000000000 000664

8. Vu D, Ruggiero M, Choi WS, Masri D, Flyer M, Shyknevsky I, Stein EG (2020) Three unsuspected CT diagnoses of COVID19. Emerg Radiol 27 (3):229-232. https://doi.org/10.1007/s1014 0-020-01775-4

9. Dane B, Brusca-Augello G, Kim D, Katz DS (2020) Unexpected Findings of Coronavirus Disease (COVID-19) at the Lung Bases on Abdominopelvic CT. AJR Am J Roentgenol:1-4. https://doi. org/10.2214/ajr.20.23240 
10. Siegel A, Chang PJ, Jarou ZJ, Paushter DM, Harmath CB, Arevalo JB, Dachman A (2020) Lung Base Findings of Coronavirus Disease (COVID-19) on Abdominal CT in Patients With Predominant Gastrointestinal Symptoms. AJR Am J Roentgenol:1-3. https:// doi.org/10.2214/ajr.20.23232

11. Hanauer DA, Mei Q, Law J, Khanna R, Zheng K (2015) Supporting information retrieval from electronic health records: A report of University of Michigan's nine-year experience in developing and using the Electronic Medical Record Search Engine (EMERSE). J Biomed Inform 55:290-300. https://doi. org/10.1016/j.jbi.2015.05.003

12. Simpson S, Kay FU, Abbara S, Bhalla S, Chung JH, Chung M, Henry TS, Kanne JP, Kligerman S, Ko JP, Litt H (2020) Radiological Society of North America Expert Consensus Statement on Reporting Chest CT Findings Related to COVID-19. Endorsed by the Society of Thoracic Radiology, the American College of Radiology, and RSNA. J Thorac Imaging. https://doi.org/10.1097/ rti.0000000000000524

13. Pan L, Mu M, Yang P, Sun Y, Wang R, Yan J, Li P, Hu B, Wang J, Hu C, Jin Y, Niu X, Ping R, Du Y, Li T, Xu G, Hu Q, Tu L (2020) Clinical Characteristics of COVID-19 Patients With Digestive Symptoms in Hubei, China: A Descriptive, Cross-Sectional, Multicenter Study. Am J Gastroenterol 115 (5):766-773. https:// doi.org/10.14309/ajg.0000000000000620
14. Bhayana R, Som A, Li MD, Carey DE, Anderson MA, Blake MA, Catalano O, Gee MS, Hahn PF, Harisinghani M, Kilcoyne A, Lee SI, Mojtahed A, Pandharipande PV, Pierce TT, Rosman DA, Saini S, Samir AE, Simeone JF, Gervais DA, Velmahos G, Misdraji J, Kambadakone A (2020) Abdominal Imaging Findings in COVID-19: Preliminary Observations. Radiology:201908. https ://doi.org/10.1148/radiol.2020201908

15. Goldberg-Stein S, Fink A, Paroder V, Kobi M, Yee J, Chernyak V (2020) Abdominopelvic CT findings in patients with novel coronavirus disease 2019 (COVID-19). Abdom Radiol (NY) 45 (9):2613-2623. https://doi.org/10.1007/s00261-020-02669-2

16. Rinaldi MF, Bartalena T, Giannelli G, Rinaldi G, Sverzellati N, Canini R, Gavelli G (2010) Incidental lung nodules on CT examinations of the abdomen: prevalence and reporting rates in the PACS era. Eur J Radiol 74 (3):e84-88. https://doi.org/10.1016/j. ejrad.2009.04.019

Publisher's Note Springer Nature remains neutral with regard to jurisdictional claims in published maps and institutional affiliations. 